Vol. 4, $\mathrm{N}^{\circ} 1$., p.67- 80, enero - marzo, 2020

\title{
Claves para la transformación digital de las pymes
}

\section{Keys for digital transformation of pymes}

Marcos Junior Muñoz Moreira. ${ }^{1}$, María Cristina Vaca Ortega. ${ }^{2}$, Elvin Brandon Mina Palacios. ${ }^{3}$ \& Mario Santiago Torres Panezo. ${ }^{4}$

DOI: https://doi.org/10.33262/visionariodigital.v4i1.1101

\begin{abstract}
The digital transformation of an pymes today is one of the processes that arouses most interest in most business sectors. According to a market research study for BBVA, published in 2017, 60\% of Spanish companies consider digitalization essential to obtain greater benefits and more success in their businesses.

However, the term digital transformation is often confused and entrepreneurs believe that it implies little more than using Social Networks or having a web page. What is clear is that you have to adapt to the new form of consumption and get where our customer is. If we are not at the same level, it will be difficult to stand out and make a place among the competition, more and more it is more prepared and constantly updated.

The first thing you should know is what exactly the digital transformation is, that is, when we talk about a process that involves not only the managers of the organization but each of the departments and employees, which you should get involved to the fullest. In general terms, it consists of taking advantage of new technologies to increase the efficiency and productivity of our business and find new opportunities aimed at business success.
\end{abstract}

Keywords: Digital transformation, organization, productivity, efficiency, competition

\section{Resumen}

La transformación digital de una pyme en la actualidad es uno de los procesos que más interés despierta en la mayoría de sectores empresariales. Según un estudio de market

\footnotetext{
Universidad Técnica Luis Vargas Torres de Esmeraldas Sede Santo Domingo, marco.munoz@utelvt.edu.ec

2 Universidad Técnica Luis Vargas Torres de Esmeraldas Sede Santo Domingo, maria.vaca@utelvt.edu.ec

3 Universidad Técnica Luis Vargas Torres de Esmeraldas Sede Santo Domingo, elvin.mina@utelvt.edu.ec

4 Universidad Técnica Luis Vargas Torres de Esmeraldas Sede Santo Domingo, mario.torres@utelvt.edu.ec
} 
research para el BBVA, publicado en 2017, el 60\% de las empresas españolas consideran imprescindible la digitalización para obtener mayores beneficios y más éxito en sus negocios.

Sin embargo, muchas veces se confunde el término de transformación digital y los empresarios creen que implica poco más que usar las redes sociales o tener una página web. Lo que está claro es que hay que adaptarse a la nueva forma de consumo y llegar allí donde se encuentra nuestro cliente. Si no estamos en su mismo nivel, será difícil destacar y hacerse un hueco entre la competencia, cada vez más está más preparada y que se actualiza constantemente.

Lo primero que debes saber es qué es exactamente la transformación digital, es decir, cuando hablamos de un proceso que implica no sólo a los directivos de la organización sino a cada uno de los departamentos y empleados, que debes involucrarse al máximo. En términos generales, consiste en aprovechar las nuevas tecnologías para aumentar la eficiencia y productividad de nuestro negocio y encontrar nuevas oportunidades encaminadas al éxito empresarial.

Palabras claves: Transformación digital, organización, productividad, eficiencia, competencia.

\section{Introducción}

En la actualidad transformación digital es un contenido muy solicitado y aplicado en la tecnología actual, lo cual ha venido actualizándose de manera rápida y ha sido un objeto de debate en los últimos años (Aguiló, 2019). En estos últimos años se ha pensado que el nacimiento de la tecnología o la digitalización del mundo era un proceso común y que no tendría significancia en mundo empresarial había preocupación se creía que no iba cubrir las necesidades digitales modernas o almacenamiento digital (Bustos, 2009).

Cuando las empresas se dieron cuenta de que podían aprovechar los nuevos datos digitalizados, empezaron a desarrollar procesos para llevarlo a cabo. Desde entonces, el ritmo de la evolución tecnológica ha aumentado exponencialmente, con lo que la capacidad de adaptación y competitividad se han vuelto aún más importantes (Krogerus \& Roman, 2011).

El paso al ecosistema digital se ha convertido en una necesidad para las empresas, ya que las nuevas herramientas tecnológicas ofrecen un valor diferencial ante la alta competitividad. 
Por ello, os ofrecemos una serie de recomendaciones para una transformación digital exitosa. (Serrano, 2017)

La transformación digital es un proceso que integra la tecnología digital en todos los aspectos del negocio y que requiere de cambios fundamentales en el ámbito de la tecnología, la cultura, las operaciones y la entrega de valor (Marín \& Muñoz Moreno, 2018).

Para aprovechar mejor las tecnologías emergentes y su rápida expansión en las actividades humanas, las empresas deben reinventarse y transformar radicalmente todos sus procesos y modelos. La transformación digital requiere un cambio de enfoque al perímetro de la empresa y centros de datos más ágiles que puedan de respaldar ese entorno. También implica mudar la tecnología anterior, que puede ser costosa de mantener para la empresa, y modificar la cultura empresarial de manera que respalde la aceleración que trae consigo la transformación digital (Marcel Planellas \& Muni, 2015).

Lo primero que tienes que tener claro es qué quieres conseguir convirtiendo tu negocio en un negocio digital. ¿Por dónde empezar? Al fin y al cabo, lo más importante es mejorar la experiencia de cliente (López, 2019). Es recomendable empezar con pequeños procesos, mediante proyectos pilotos, para ajustar tus objetivos. (Molina, 2019). Datisa ha elaborado un documento donde aconseja que es necesario una formación digital para que puedan entender la magnitud del cambio. Es claro que las TIC ya forman parte, desde hace años, de nuestro mundo digital; pero con tener modernas y potentes herramientas tecnológicas no basta. La transformación digital en las empresas es uno de los principales focos de actuación para la mayoría de los gestores hoy día. Ya sean microempresarios, pequeños, medianos o grandes empresarios (Mireles, 2009).

Pero antes de hablar de un plan de plan de transformación digital, recordemos qué es la transformación digital:

La transformación digital es el proceso a través del cual una empresa incorpora nuevas tecnologías y procesos digitales a su sistema de actividades. El objetivo de este proceso es evolucionar la empresa para que sea más eficiente y que se adapte a su entorno. La transformación digital es un cambio que se está produciendo de manera rápida pero progresiva. Una transformación de la sociedad fruto de la adopción de la tecnología digital en las actividades diarias de las personas (Naranjo, 2017). 
Las pymes han optado por reemplazar sus interacciones tradicionales por la trasformación digital impulsada a mejora su estructura empresarial comprometiéndose a nuevos retos de mercado. Para alguna empresa, que aún no toman la iniciativa debido a políticas y desactualización de los departamentos, ellos saben que deben someterse a este cambio si desean sobrevivir en el mundo empresarial (González, 2007).

Una vez que se dan estos cambios los consumidores esperan que los cambios sean productivos en todos los ámbitos, caso contrario no tendrán oportunidad de sobrevivir en el mundo digital (Betancourt, 2009).

Los procesos de trasformación digital no solo dependen de las empresas, tiene que ver también con el comportamiento del consumidor originario por su aparición y manejo de nuevas tecnologías, esta aparición de mundo de la digitalización está afectando a las empresas multidimensionales y las pymes, estas entidades no escapan de esta realidad. Día a día con mayor frecuencia están percibiendo el proceso de transformación digital como alternativa costosa, pero de no involucrarse o asumirla podrán dejar de ser competitivas o incluso salir del mercado.

Es decir que las pymes, esta obligadas en la actualidad asumir el proceso de transformación digital, como un elemento estratégico hacia una innovación descriptiva, que algunas organizaciones ya han emprendido este cambio y los resultados fueron exitosamente buenas.

Las empresas tradicionales si están obligadas a contar con un plan de transformación digital de los negocios, requiriendo esta transformación de un enfoque, una estrategia y un cambio cultural global en las organizaciones.

Esta época digital se ha convertido en un factor clave en un marco de variedad que favorece el pensamiento creativo, analítico, esta nueva experiencia del cliente digital está obligando a reestructurar los proceso y los canales de comunicación como el mundo moderno a los consumidores para lograr a un corto plazo obtener su fidelidad.

Plantear un proceso de transformación digital para las Pymes para diferentes fines, el factor humano en indispensable para poder consolidarlo es decir trabajar para la humanización de la humanidad como lo propone Edgar Morín (1999), en su planteamiento de los siete saberes, publicado por la UNESCO. Considerando que debemos tener en cuenta ese esquema se podrá en contratar un sentido humano y social acerca de la transformación digital y acceso a los productos desde sus hogares o lugares de trabajo. 
ISSN: 2602-8506

Vol. 4, $\mathrm{N}^{\circ} 1 .$, p.67- 80, enero - marzo, 2020

La transformación digital no debe de estar alejado de las grandes empresas los cambios que se dan en el consumidor por la aparición de las grandes tecnologías afecta de una u otra manera a las empresas multidimensional y la pymes que no están tan alejados de la realidad. No obstante, con frecuencia las pymes perciben los procesos de una transformación digital como algo inalcanzable, pero corren el riesgo de no asumirlo podrán de ser empresa de competencia e incluso dejar de funcionar hasta salir del mercado.

Por esta razón las pymes deben asumir el proceso de transformación digital, como un elemento estratégico hacia una innovación disruptiva, que algunas organizaciones ya han emprendido exitosamente.

Las empresas tradicionales están obligadas a contar con un plan de transformación digital de los negocios, requiriendo esta transformación de un enfoque, una estrategia y un cambio cultural global en las organizaciones.

En esta era digital, la colaboración se ha convertido en un factor clave, en un marco de diversidad que favorece el pensamiento creativo. Esta nueva experiencia del cliente digital obliga a que se dé una reestructurar procesos y las vías de comunicación con los mismos canales de venta, y las formas de lograr fidelizarles.

El proceso de transformación digital no es exclusivo de las grandes empresas existentes sino s sino de los cambios en el comportamiento del consumidor originados por la aparición de nuevas tecnologías, están afectando a las empresas multinacionales y las pymes no escapan de este proceso de la digitalización; no hay que desprendernos de que con frecuencia las pymes están percibiendo los procesos transformación digital a nivel del mundo, como una tarea costosa, pero de no asumirla podrán dejar de ser competitivas e incluso salir del mercado. Por esta razón las pymes deben asumir el proceso de la digitalización, como un elemento estratégico hacia una innovación disruptiva, que algunas organizaciones ya han emprendido exitosamente. Las empresas están obligadas a contar con un plan de transformación digital de sus negocios, requiriendo esta transformación de un enfoque, una estrategia y un cambio cultural global en las organizaciones. En esta era digital, la colaboración se ha convertido en un factor clave, en un marco de diversidad que favorece el pensamiento creativo. La nueva experiencia del cliente digital está obligando a reestructurar procesos y los canales de comunicación con los mismos, los canales de venta, y las formas de lograr fidelizarles. 
Del mismo modo las empresas que deciden involucrarse en a los nuevos cambios puedan estar preparados y capacitados para brindar un servicio profesional y responsable para que tenga un éxito a corto plazo en todos sus ámbitos (Gómez, 2011).

Es decir que transformación digital es enfrentarse a un cambio total donde las empresas puedan asumir retos y descubrir la modernización par que estén en constante evolución, así como lo hace la tecnología (Schwab, 2017).

\section{Metodología}

Desde hace ya unos años estamos asistiendo, casi sin darnos cuenta, a una verdadera revolución en el uso de las nuevas tecnologías en nuestro día a día. Ahora es difícil no ver por la calle a una persona sin su smartphone, tablet o pulsera digital. Y en nuestras casas ya es habitual la fibra óptica o los aparatos domésticos conectados a internet. Lo digital invade nuestras vidas, pero, ¿qué sucede en las empresas?

Las organizaciones se han ido incorporando poco a poco al mundo digital, empezando por lo más obvio, como el uso de internet en las comunicaciones (web, correos electrónicos, calendarios compartidos), y la gestión integrada de sus procesos más habituales, como son los administrativos y financieros. Es el primer nivel de digitalización de una organización. A partir de este punto la empresa está en condiciones de dotar de mayor complejidad a sus procesos, que le permitirán no solo automatizarlos, sino abordar nuevas capacidades que le ayuden en su estrategia corporativa. Tres cuartas partes de los directivos españoles (más del 75\%) afirman que la transformación digital será una palanca de cambio en sus organizaciones, a pesar de que en nuestro país los datos muestran que solamente un tercio de las compañías están preparadas. La presente investigación es de carácter de cualitativa y cuantitativa, la cual se fundamentó en el análisis de los resultados de las encuestas realizadas sobre claves para la transformación digital de las pymes.

La transformación digital puede implicar la reelaboración de los productos, procesos y estrategias dentro de la organización mediante el aprovechamiento de la tecnología digital. Como tal, requiere un examen y reinvención de la mayoría, si no de todas las áreas dentro de una organización, de su cadena de suministro y flujo de trabajo, las habilidades de sus empleados, así como procesos de discusión a nivel de junta directiva, interacciones con clientes y su valor para las partes interesadas. Ayuda a una organización a seguir el ritmo a las demandas emergentes de los clientes, manteniéndolas en el futuro. Es así que la digitalización en la actualidad permite que las 
ISSN: 2602-8506

organizaciones compitan en un entorno económico y estén en constante actualización a medida que avanza la tecnología, esto en con el fin de involucrase directamente con la transformación digital cabe indicar que para cualquier empresa u organización sin fines de lucro o institución que busquen sobrevivir.

\section{Resultados}

A continuación, se detalla el análisis de los resultados obtenidos de la encuesta y entrevista aplicada a 100 personas entre aquellos empleados de diversas instituciones, y estudiantes universitarios en administración de empresas, la encuesta realizada trató sobre el conocimiento de la transformación digital de las pymes.

Tabla 1. Encuesta

\begin{tabular}{|c|c|c|c|c|c|}
\hline & & & Estadís & ticos & \\
\hline & & $\begin{array}{l}\text { ¿Tiene usted conoci- } \\
\text { miento sobre que es } \\
\text { la transformación di- } \\
\text { gital? }\end{array}$ & $\begin{array}{l}\text { ¿Crees que es impor- } \\
\text { tante la implementación } \\
\text { de la tecnología de los } \\
\text { procesos (Administrati- } \\
\text { vos, comerciales...) en } \\
\text { una empresa? }\end{array}$ & $\begin{array}{l}\text { ¿Qué te llevaría a tomar } \\
\text { la decisión de iniciar un } \\
\text { proceso de transforma- } \\
\text { ción digital en una em- } \\
\text { presa? }\end{array}$ & $\begin{array}{l}\text { ¿Conoce usted el } \\
\text { significado PY- } \\
\text { MES? }\end{array}$ \\
\hline & Válido & 100 & 100 & 100 & 100 \\
\hline & Perdidos & 0 & 0 & 0 & 0 \\
\hline
\end{tabular}

Elaborado por: Grupo de investigación.

Tabla 2. ¿Tiene usted conocimiento sobre que es la transformación digital?

\begin{tabular}{llllll}
\hline & & Frecuencia & Porcentaje & $\begin{array}{l}\text { Porcentaje } \\
\text { válido }\end{array}$ & $\begin{array}{l}\text { Porcentaje } \\
\text { acumulado }\end{array}$ \\
\hline \multirow{3}{*}{ Válido } & No & 9 & 9 & 9 & 9 \\
& Si & 61 & 61 & 61 & 70 \\
& Tal vez & 30 & 30 & 30 & 100 \\
& Total & 100 & 100 & 100 & \\
\hline
\end{tabular}

Elaborado por: Grupo de investigación.

De acuerdo con la encuesta realizada nuestra primera pregunta tuvo como resultados de un $9 \%$ los cuales respondieron que no tienen conocimiento sobre la transformación digital, y el $61 \%$ si conocen sobre el tema en cuestión, sin embargo, un 30\% de las personas no están seguras sobre que es realmente la Transformación digital. 
WWW.visionariodigital.org

Vol. 4, $\mathrm{N}^{\circ} 1$., p.67- 80, enero - marzo, 2020

El desconocimiento, el miedo al cambio y los mitos en torno a la transformación digital hacen que a muchos empresarios se les ponga la piel de gallina cuando escuchan hablar de este tema. La consecuencia es que muchas organizaciones continúan con procesos de trabajo obsoletos y poco rentables.

La digitalización ofrece un enorme potencial para ganar competitividad en un mundo cada vez más tecnológico. Así lo demuestra un estudio del Massachusetts Institute of Technology, que afirma que las empresas más tecnológicas son más rentables y tienen clientes más satisfechos.

Tabla 3. ¿Crees que es importante la implementación de la tecnología de los procesos (administrativos, comerciales...) en una empresa?

\begin{tabular}{cccccc}
\hline & Frecuencia & Porcentaje & $\begin{array}{l}\text { Porcentaje } \\
\text { válido }\end{array}$ & $\begin{array}{c}\text { Porcentaje } \\
\text { acumulado }\end{array}$ \\
\hline \multirow{3}{*}{ Válido } & No & 3 & 3 & 3 & 3 \\
& $\mathrm{Si}$ & 80 & 80 & 80 & 83 \\
& Tal vez & 17 & 17 & 17 & 100 \\
& Total & 100 & 100 & 100 &
\end{tabular}

Elaborado por: Grupo de investigación.

Sobre la importancia de la implementación de la tecnología de los procesos en una empresa obtuvimos un 3\% los cuales respondieron que no era importante este tipo de la implementación en las empresas, sin embargo, liderando la tabla de resultados con un $80 \%$ con respuesta positiva para implementar la tecnología en los procesos empresariales, pero también obteniendo como respuesta de "talvez" de un $17 \%$.

La tecnología impulsa la necesidad de la transformación digital y respalda la digitalización de una organización. Además, no existe una sola aplicación o tecnología que permita la transformación digital, sino que existen múltiples procesos clave que una organización generalmente debe tener para afrontar la transformación:

La computación en nube, por ejemplo, le otorga a la organización un acceso más rápido al software que necesita, nuevas funcionalidades y actualizaciones, además de almacenamiento de datos, y le permite ser lo suficientemente ágil como para transformarse. 
ISSN: 2602-8506

www.visionariodigital.org

Vol. 4, N¹., p.67- 80, enero - marzo, 2020

La tecnología de información permite a una organización enfocar su inversión de talento y el dinero destinado a investigación y desarrollo en soluciones personalizadas que respalden sus requisitos y los procesos que lo diferencian en el mercado.

Las plataformas móviles permiten que el trabajo se realice donde sea y cuando sea; Machine Learning y las tecnologías de inteligencia artificial brindan a las organizaciones conocimientos para tomar decisiones más precisas sobre ventas, marketing, desarrollo de productos y otras áreas estratégicas

Tabla 4. ¿Qué te llevaría a tomar la decisión de iniciar un proceso de transformación digital en una empresa?

\begin{tabular}{|c|c|c|c|c|c|}
\hline & & Frecuencia & Porcentaje & $\begin{array}{l}\text { Porcentaje } \\
\text { válido }\end{array}$ & $\begin{array}{l}\text { Porcentaje } \\
\text { acumulado }\end{array}$ \\
\hline \multirow{4}{*}{ Válido } & $\begin{array}{l}\text { Presión de la competencia. Están } \\
\text { más avanzados. }\end{array}$ & 78 & 78 & 78 & 78 \\
\hline & Presión de los Clientes. & 15 & 15 & 15 & 15 \\
\hline & Presión interna de la propia empresa. & 7 & 7 & 7 & 100 \\
\hline & Total & 100 & 100 & 100 & \\
\hline
\end{tabular}

Elaborado por: Grupo de investigación

De acuerdo con la pregunta planteada sobre la decisión de iniciar un proceso de transformación digita, un 78\% respondió que es la presión de la competencia. Están más avanzados, un 15\% por presión de los clientes, y solo un 7\% por presión interna de la propia empresa.

Tabla 5. ¿Conoce usted el significado PYMES?

\begin{tabular}{llllll}
\hline & & Frecuencia & Porcentaje & $\begin{array}{l}\text { Porcentaje } \\
\text { válido }\end{array}$ & $\begin{array}{l}\text { Porcentaje } \\
\text { acumulado }\end{array}$ \\
\hline \multirow{3}{*}{ Válido } & No & 35 & 35 & 35 & 35 \\
& $\mathrm{Si}$ & 65 & 65 & 65 & 100 \\
& Total & 100 & 100 & 100 & \\
\hline
\end{tabular}

Elaborado por: Grupo de investigación.

De acuerdo con nuestra investigación decidimos preguntar a las personas sobre cuál es el significado de las pymes, recopilando sus respuestas obtuvimos un 35\% de las personas 
ISSN: 2602-8506

WWW.visionariodigital.org

Vol. 4, $\mathrm{N}^{\circ} 1$., p.67- 80, enero - marzo, 2020

encuestadas no saben el significado de Pymes, pero con mayor acotación obtuvimos un 65\% de personas que si saben lo que son las pymes.

Pymes quiere decir pequeñas y medianas empresas. En el país, se llama Pymes al conjunto de pequeñas y medianas empresas, que, de acuerdo al número de trabajadores, volumen de ventas, años en el mercado, y sus niveles de producción, activos, pasivos (que representan su capital) tienen características similares en sus procesos de crecimiento.

Tabla 6. Entrevista realizada a un supervisor del área de mecánica.

\begin{tabular}{|c|c|c|}
\hline PREGUNTA & RESPUESTA & ANALISIS \\
\hline $\begin{array}{l}\text { ¿Por qué es importante para } \\
\text { usted la transformación digital } \\
\text { de las PYMES? }\end{array}$ & $\begin{array}{l}\text { La transformación de las actividades, pro- } \\
\text { cesos y modelos empresariales para aprove- } \\
\text { char al máximo las oportunidades de una } \\
\text { serie de tecnologías digitales de forma es- } \\
\text { tratégica en todos los aspectos de los nego- } \\
\text { cios. }\end{array}$ & $\begin{array}{l}\text { La transformación digital ha sido el sector de } \\
\text { los negocios y las empresas. Las herramien- } \\
\text { tas y plataformas digitales, y las diferentes } \\
\text { formas en que las usamos en nuestra vida } \\
\text { personal, laboral y social, han cambiado para } \\
\text { siempre cómo funcionan las empresas. }\end{array}$ \\
\hline $\begin{array}{l}\text { ¿Cómo ayuda la transforma- } \\
\text { ción digital en su empresa? }\end{array}$ & $\begin{array}{l}\text { Con los cambios constantes en las diferen- } \\
\text { tes industrias y el uso de todo tipo de herra- } \\
\text { mientas tecnológicas para optimizar los } \\
\text { procesos internos de las empresas, es una } \\
\text { necesidad integrar estas ayudas para ser } \\
\text { mucho más competitivo. }\end{array}$ & $\begin{array}{l}\text { Ampliar sus ecosistemas de gestión de la in- } \\
\text { formación y mejorar sus procesos internos. }\end{array}$ \\
\hline $\begin{array}{l}\text { ¿En qué tipo de áreas se utiliza } \\
\text { más la transformación digital? }\end{array}$ & $\begin{array}{l}\text { A nivel empresarial un } 95 \% \text { de nuestra em- } \\
\text { presa cuenta con transformación digital ya } \\
\text { que facilita la realización del producto y el } \\
\text { manejo administrativo de la empresa. }\end{array}$ & $\begin{array}{l}\text { La transformación digital es de vital impor- } \\
\text { tancia en la actualidad ya que no ayuda en la } \\
\text { productividad de la empresa. }\end{array}$ \\
\hline $\begin{array}{l}\text { ¿Cómo capacitamos a los tra- } \\
\text { bajadores para que se adapten } \\
\text { a este cambio organizacional? }\end{array}$ & $\begin{array}{l}\text { Primero se les avisa con anticipación a } \\
\text { nuestros trabajadores para que estén abier- } \\
\text { tos al cambio, también ayudamos por me- } \\
\text { dio de charlar tanto motivacionales como } \\
\text { educadoras. }\end{array}$ & $\begin{array}{l}\text { El resultado se traduce en una mayor flexibi- } \\
\text { lidad laboral, conciliación familiar y en la } \\
\text { gestación de un sistema centralizado de in- } \\
\text { formación que reúna el conocimiento y } \\
\text { aprendizaje compartido de la empresa }\end{array}$ \\
\hline $\begin{array}{l}\text { ¿Cuáles son sus claves para } \\
\text { una buena transformación di- } \\
\text { gital? }\end{array}$ & $\begin{array}{l}\text { El empleo de tecnologías que permiten un } \\
\text { marketing moderno que reinvente la expe- } \\
\text { riencia de compra y del servicio al cliente, } \\
\text { son algunas de las claves para dicha trans- } \\
\text { formación. }\end{array}$ & $\begin{array}{l}\text { Es importante que el proceso innovación tec- } \\
\text { nológica esté respaldado por un asesor tec- } \\
\text { nológico que ayude a comprender las necesi- } \\
\text { dades reales de digitalización y a implemen- } \\
\text { tarlas de manera coherente y responsable de } \\
\text { acuerdo con las prioridades, objetivos y ca- } \\
\text { pacidades de inversión de la organización. }\end{array}$ \\
\hline
\end{tabular}

Elaborado por: Grupo de Investigación 


\section{Discusión}

De acuerdo con los resultados obtenidos en la aplicación de las encuestas y entrevistas a las claves para la transformación digital de las Pymes, el reto de la transformación digital es antes un reto de gestión que tecnológico, y en temas de gestión ya hay mucha sabiduría acumulada sobre cómo gestionar la innovación, la calidad, la internacionalización, la organización del trabajo.

La transformación digital en organizaciones o empresas es el proceso a través del cual incorporamos la tecnología a nuestra entidad para poder adaptarla al entorno, siempre desde una visión estratégica y una cultura digital. No se trata sólo de comenzar a trabajar las redes sociales, tener una web innovadora, poner en marcha un comercio o digitalizar todos los documentos físicos que tenemos en la oficina.

Cuando hablamos de un proceso de transformación digital en la entidad tenemos que entender una reorganización del modelo de trabajo. Plantear nuevas formas de trabajar y de incorporar y asimilar en nuestros procesos nuevas metodologías de funcionamiento.

Así mismo podemos deducir que los resultados de esta investigación demuestran que la Transformación digital de las Pymes es importante para el crecimiento empresarial a nivel nacional e internacional y con ello podemos deducir que para garantizar el éxito de un proceso de transformación digital es necesario diseñar una planificación para su implementación. Tenemos que seguir una serie de pasos, que nos ayudan a impulsar estos procesos.

Es importante, antes del diseño del plan de acción, analizar y definir objetivos. Y sin duda, la formación será clave en todo el proceso.

\section{Conclusiones}

- Los resultados de esta investigación demuestran que las claves para la transformación digital son importantes dentro de una empresa. El hecho digital provoca muchos cambios y, además de los tecnológicos y empresariales, se debe empezar a poner el foco en los aspectos sociales. Como la empleabilidad. El avance de la tecnología permitirá a las empresas automatizar los procesos más complejos y convertirlo en algo más sencillo y así evitar gastos innecesarios en esos procesos. Esta característica permitirá que la empresa agilite Esta característica permite que las empresas se vuelvan más ágiles y puedan utilizar su capital humano de forma más eficaz. 
ISSN: 2602-8506

WWW.visionariodigital.org

Vol. 4, $\mathrm{N}^{\circ} 1$., p.67- 80, enero - marzo, 2020

En la actualidad los clientes son partes importantes de una empresa lo cual deben ser escuchado y cubran las necesidades específicas, con el avance de la tecnología se trata de aprovechar en las empresas, los procesos manuales y ubicarles en datos digitales, es decir que las empresas están obligada a adoptar nuevas tecnologías a medidas que se dispongan en ella con el fin de mirar al futuro. Y sacra buenos resultados en todo el ámbito económico, social, organizacional.

- Al iniciar el proceso de transformación digital, es indispensable que la empresa se plantee cómo quiere que sea su evolución y qué tecnologías puede aprovechar para lograr su objetivo. Si bien las empresas conocen la urgencia de fomentar la transformación digital desde el interior, se omiten variables directivas que permitan la continuidad de las estrategias. Estos cambios afectan todos los procesos de la cadena de valor de la organización, por esta razón es importante contar con diferentes perspectivas gerenciales que lleven adelante escenarios de proyección empresarial acorde a los objetivos organizacionales.

\section{Referencias bibliográficas}

Aguiló, Jaume Martínez. Industria 4.0. Santiago: Editorial CEPAL, 2019.

Betancour, Fernando Jaramillo. Valoración de empresas. Ecoe Ediciones, 2009.

Bustos, Rodrigo Zúñiga. PYMES. El Cid Editor | apuntes, 2009.

Gómez, Graciela Lara. Gestión de empresas sociales. Editorial Miguel Ángel Porrúa, 2011.

González, Francisco Caro. Gestión de empresas informativas. McGraw-Hill España, 2007.

KROGERUS, MIKAEL, y TSCHÄPPELER ROMAN. «El pequeño libro de las grandes decisiones.» En El pequeño libro de las grandes decisiones, de MIKAEL KROGERUS y TSCHÄPPELER ROMAN, 176. Barcelona: ALIENTA, 2011.

López, David. Gestión de programas de proyectos informáticos (y no informáticos). Editorial UOC, 2019. 
SSN: 2602-8506

www.visionariodigital.org

Vol. 4, $\mathrm{N}^{\circ} 1$, p.67- 80, enero - marzo, 2020

Marcel Planellas, y Anna Muni. «El libro de las decisiones estratégicas.» en El libro de las decisiones estratégicas, de Marcel Planellas y Anna Muni, 216. España, Madrid: conecta, 2015.

Marín, Jesús Martínez, y José Luis Muñoz Moreno. Aprender en las organizaciones de la era digital. Editorial UOC, 2018.

Mireles, Norberto C. González. Empresas pequeñas, empresarios grandes. El Cid Editor|apuntes, 2009.

Molina, Gerardo. El negocio de la pasión y sus claves. Wanceulen Editorial, 2019.

Naranjo, Francisco. «Transformación Digital.» En Transformación Digital, de Francisco Naranjo, 230. México: Observatorio. Digital, 2017.

Schwab., Klaus. «La cuarta revolución industrial.» En La cuarta revolución industrial, de Klaus Schwab., 325. Madrid: EDT, 2017.

Serrano, Pipo. La transformación digital de una redacción y el periodismo móvil (mojo). Editorial UOC, 2017.

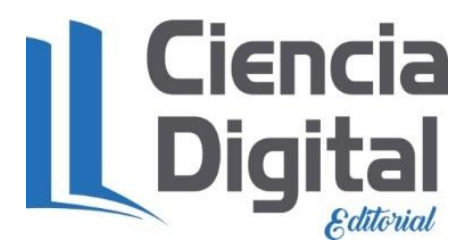


ISSN: 2602-8506

Vol. 4, $\mathrm{N}^{\circ} 1$., p.67- 80, enero - marzo, 2020

\section{PARA CITAR EL ARTÍCULO INDEXADO}

Muñoz Moreira, M. J., Vaca Ortega, M. C., Mina Palacios, E. B., \& Torres Panezo, M. S. (2020).

Claves para la transformación digital de las pymes. Visionario Digital, 4(1), 67-80. https://doi.org/10.33262/visionariodigital.v4i1.1101

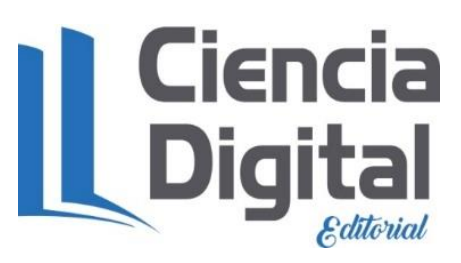

El artículo que se publica es de exclusiva responsabilidad de los autores y no necesariamente reflejan el pensamiento de la Revista Visionario Digital.

El artículo queda en propiedad de la revista y, por tanto, su publicación parcial y/o total en otro medio tiene que ser autorizado por el director de la Revista Visionario Digital.
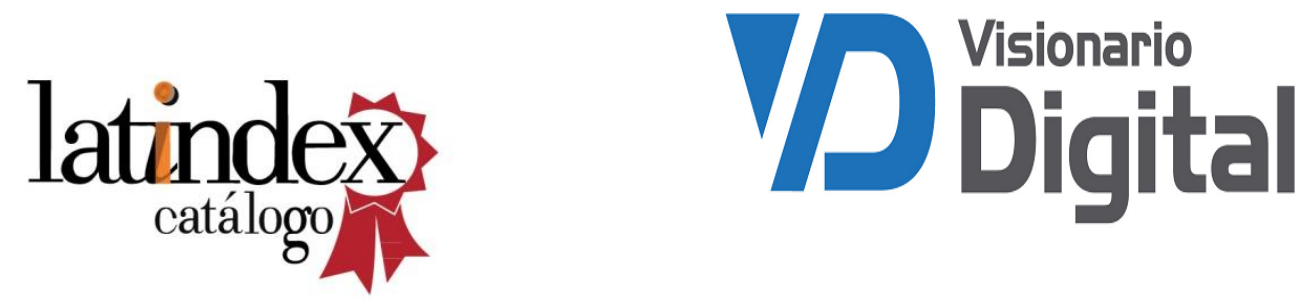